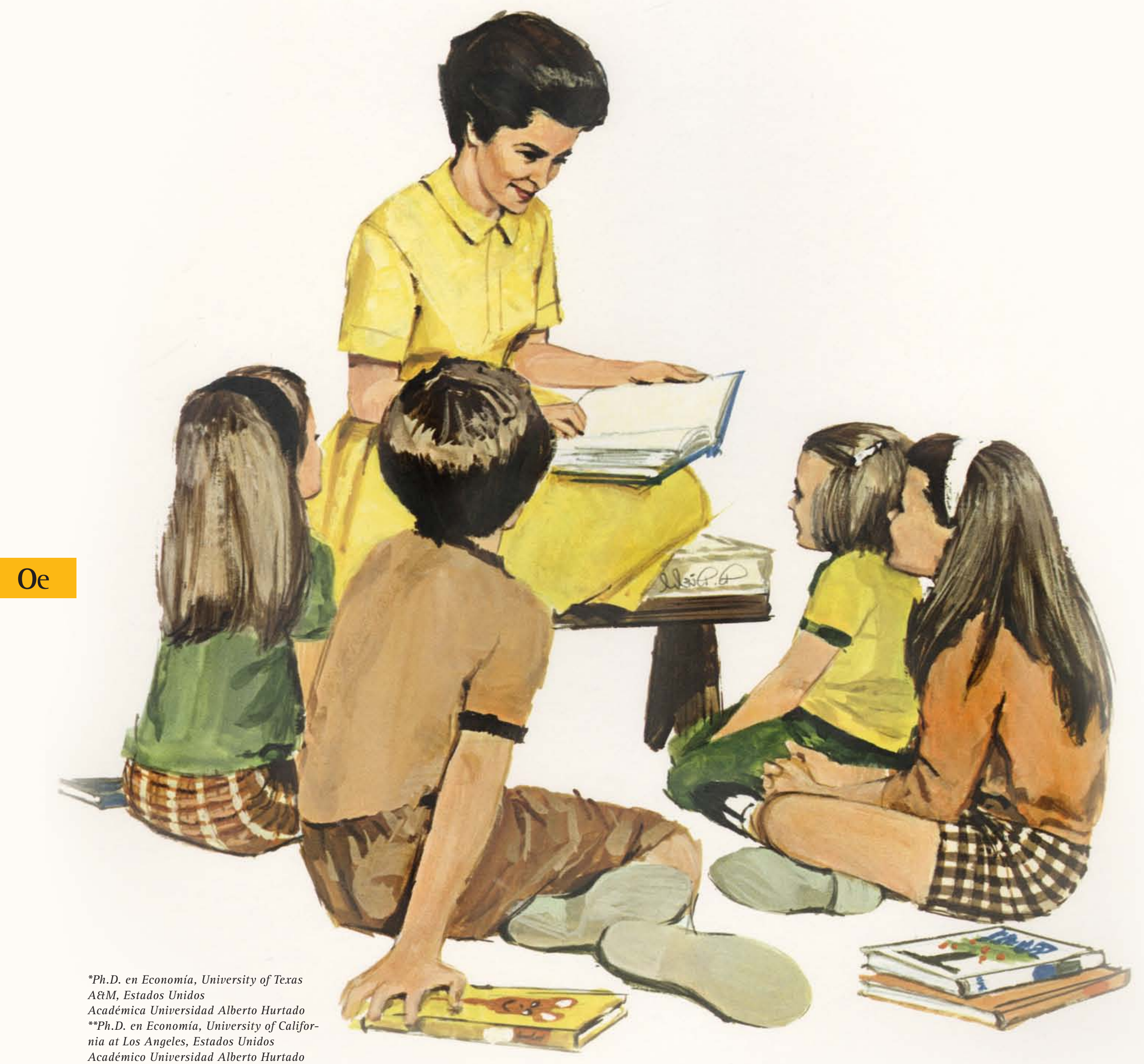

\title{
La (olvidada) carrera docente
}

Por Marcela Perticara ${ }^{*}$ Carlos Ponce*

La educación, como industria, se organiza verticalmente: los alumnos de educación factor usado para 'producir' conocimientos y habilidades de nivel universitario. Esta or ganización jerarquica de la industria educativa tiene, al menos, dos consecuencias económicas básicas. La primera, que la cantidad de capital humano, medida por el número de personas, decrece a medida que avanzamos en el nivel educativo. En otras palabras el numero de personas con educacion básica es siempre mayor al num mero de individuos con educacion mediay estos ult mos superan en numero a los individuos con destrezas unversitarias. La segunda, que la calldad de capital humano a la que puede aspiranse

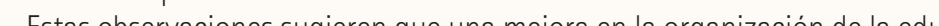

Es y media tien un potencia impacto mocóm similares en el sector educativo superior. Sin embargo, el debate núblico se ha centrado con inusitada intensidad en este último eslabón y poco, o nada, se discute sobre la calidad y el financiamiento de los tramos iniciales. ¿Sugiere esta realidad que la industria sitaria? ¿Es la calidad del capital humano adquirido en los niveles básico y medio la mejor posible? Es razonable creer que el sistema actual conduce a que alumnos de similares habilidades cognitivas finalicen su formación media con similar calidad de capital humano independientemente de nivel socioeconomico de origen?

La evidencia disponible sugiere que nuestra industria educativa está lejos de funcionar adecuadamente. Si bien, como señalan Hsieh y Urquiola', con las reformas de los años 80 (financiamiento público per cápita y municipalización de los colegios públicos), se logró una rapida expansión de la oferta de ensenanza básica y med a a traves de la creación

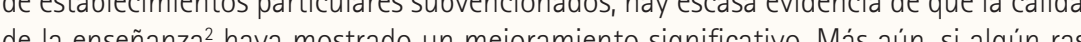
go caracteriza a nuestro sistema educativo es su magro rendimiento Indicativo de ello es que el sector privado no subvencionado ostenta indicadores de performance escola muy por debajo de los existentes en paises desarrollados para estudiantes de simitr nivel socioeconómico. A menos que aceptemos poseer desventajas cognitivas innotas, hay indicios claros de que la producción de educación está lejos de funcionar en forma eficiente y equitativa.

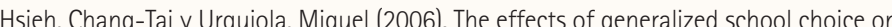
Stratification: Evidence from, Chile's voucher program Jounal of Public Economics 90 pp 1477 - 1503 Ver por ejemplo Mizala, A. Y Romaguera, P. (2001). Factores socioeconómicos explicativos de los resultados escolares en la educación secundaria en Chile, El Trimestre Económico68 (4), 515-549; Mizala, A. y Romaguera, P. (2005). Calidad de la Eaucación Chilena: el Desafio de la Proxxima Decada, en P.Meller (2012). Voucher system and school effectiveness: parental choice decision-making, Estudios de Economia 39 (2), pp. 123-141. 
Existe consenso en la literatura respec- pita (superior al promedio OECD de 1.2 veto al rol clave que los profesores y el IIderazgo directivo ejercen en la y adquisición de capital humano. $0^{3}$ Y estos razón entre el salario de un docente al inson, justamente, aspectos que han estado cio y el PIB per cápita es 0.45 , mientras que apenas incluidos en las recientes discusio- es 0.63 para el promedio de países de la nes públicas.

Si funciones docentes y directivas resul- inicial es relativamente muy baja en Chil tan esenciales para el éxito del sistema edu- lo que podría estar creando un efecto de Primero: ifantos cobuestras condiciones individuos que eligen la carera locente. aborales la selección de aquellosindividuos De esta manera, los que optan por mancon mayor talento para desempeñar es- tenerse en la profesión son los que tienen tas tareas? Segundo: ifavorecen nuestras costos de oportunidad mucho más bajos. tas tareas? Segundo. Lfavorecen nuestras temente?

En este artículo estudiamos brevemente tres aspectos determinantes de las condiciones laborales en educación. salarios docentes, horas lectivas y tamaño de clases.

INGRESOS DOCENTES Y COSTOS DE OPORTUNIDAD

Los ingresos esperados constituyen la senal econosica más importante para la

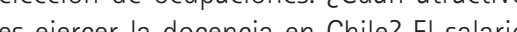
promedio de un profesor de educación básica es, en la actualidad de 530 mil pesos mensuales. La información disponible en www.mifuturo.cl muestra que el ingreso esperado de los estudiantes de pedagogia se encuentra entre 400 y 600 mil pesos mensuales, independientemente de la especialidad y/o universidad de egreso, con excepción de aquellos con especialización en matemática y computación, cuyos in-
gresos aparecen en el rango de 600-800 gresos aparecen en el rango de 600-800
mil pesos mensuales. mil pesos mensuales. 4 . Por otro lado, el
salario promedio para un egresado de salario promedio para un egresado de
educación superior de entre 25 y 65 años Ver por ejemplo, Rockofff $J$. (2004). The I Impact of Individual Teachers on Student Achievement: mic Review94 (2). Papers and Proceedings, po. 247-252. ResultadosmásmixtospresentaHarris, $D$ yT. Sass (2011), Teacher training, teacher quality and student achievement, J
Economics95 (7-8), pp 798-8 "Estimado usando la Encuesta CASEN 2009 DECD (2011), Education at a Glance 2011: OECD indicators, OECD Publishing. asciende a 800 mil pesos mensuales. En

pen

promedio, 1,5 veces superior a su ingreso.

Los profesionales de la educación en Chile tienen salarios anuales que están un 40-50\% por debajo del promedio para los paises de la OECD. Es cierto que el salario de un docente con 150 más años de antigüedad equivale a 1.5 veces el PIB per cá-
HORAS LECTIVAS

La calidad de la enseñanza depende en la habilidad del docente sino también de una serie de actividades no lectivas. esfuerzo en preparación de las clases, busqueda y selección de materia, cordinacion de distintas areas docentes. ye estas tareas son difictes de ortante Y medir, mientras que el tiempo dedicado medible. En la medida en que ambas tareas compiten entre si por la preferencia del profesor, una alta dedicación a tiemscaso esfuerzo en actividades no lectivas. Estimaciones de $O E C D^{6}$ indican que los maestros chilenos dedican a actividades ctivas unas 1.200 horas anuales, es deser Japón, Carras y escomo maximo tres Chile el promedio supera tas 5 hou

MAÑO DE CLASE

La producción de conocimientos tiene un inevitable componente de bien públi0, pero está sujeta también a efectos de salase depende del cimiento transmitido sus compañeros. Cuanto menos 'atento' pester capacitacion, etc. Desy medi, mientras que el tien de observar po docente no es más que el reflejo de un in, el $70 \%$ de su tiempo total de trabajo. . 作 congestion: la habilidad de un estudiante sea un estudiante, toda la clase se verá profesionales sino también de esfuerzo y cación en actividades no lectivas. Toclase juega entonces un rol esencial?. das las acciones para aumentar el interés Para entender concretamente el efecto de los jovenes por estudiar carreras pedatamaño, considere el siguiente ejemplo. gógicas han ido en la dirección de reducirsuponga que un estudiante cualquiera les el costo de estudiar (becas), cuando el se comporta 'adecuadamente' el 98\% del valor de seguir el camino docente depende tiempo de clase. En una clase de 21 estu- también de condiciones de trabajo: saladiantes, el tamaño promedio de clase para rios, horas de trabajo, valorización del ro menos un estudiante no se estará compor-

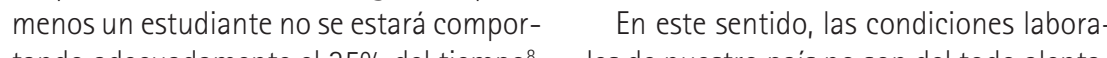
Siel tamaño de la clase es de 30 estudian- doras. La ley de carrera docente, uno de los tes, el tamaño promedio en Chile, enton- proyectos emblemáticos de este gobierno ces al menos un estudiante no se portará duerme desde hace más de un año en el como corresponde el $46 \%$ del tiempo total Congreso, siendo en teoría una de las priode clase. Si en cambio, un estudiante tu- ridades qubernamentales en materia eduviera el comportamiento esperado el 99\% cacional. Independiente de las debilidades de su tiempo, la anterior cifra se reduciria que este proyecto especifico pudiera tener, a un 26\%. La calidad del profesor condi- es necesario retomar las discusiones sobre ciona la relación entre desempeño y ta- cómo lograr formar y contar con profesomaño de clase, pero, claramente, el tipo de res más efectivos.

profesor que necesitamos para clases más numerosas es distinto al necesario para clases más pequeñas si queren

A MODO DE CONCLUSIÓN

En definitiva, independientemente de que podamos "tentar" a más jóvenes talentosos a estudiar pedagogias, es también importante atender a las condiciones de trabajo de los docentes chilenos. La "calidad" de los docentes importa, pero esta calidad es tambien endógena a la manera dentro del sistema educativo.

El ejemplo planteado en el apartado anterior muestra que un tamaño de clase adecuado es una variable importante pa obtener resultados acadénicos satisfactorios. Sin embango, reducir el tamaño de las clases es usualmente costo serí que los estudiantes se 'portasen' mejor. Hay razones para creer que el tiempo que un estudiante se comporta adecuada mente depende de la calidad del docente los mejores profesores motivan a sus estudiantes y promueven la atención en clase Sin embargo, la motivación y el lideraze docente no solo requieren de los mejores (2001). 'Educational production', Quarterly
Oe

El modelo teórico se atribuye a Lazear, E.P. numerososos estudios empiricos que ligan tamaño

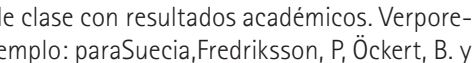
H.Oosterbeek (2013) Long-Term Effects of Class (11), pp 249-285; para Boliviva, truviola Mige 2006). Identifying Class Size Effects in Developing Countries: Evidence from Rural Bolivia,
Review of Economics and Statistics 88 (1), pp 5. (2009). Class Size Reduction and Student Teacher Quality and Class Size, Journal of Hum Resources 44(1), pp.223-250
8Este cálculo se hace suponient que los 0.98 de manera indenendiente El resultadio es entonces, la potencia de 0,98 a la 21.
Decano: Jorge Rodriguez Grasi

Fono Facultad: 28897366

fen.uahurtado.cl

Producción OE: Escuela de Periodismo UAH 